\title{
AltMM 2018 - 3rd International Workshop on Multimedia Alternate Realities
}

\author{
Teresa Chambel \\ LASIGE, Faculdade de Ciências \\ Universidade de Lisboa \\ Lisboa, Portugal \\ tc@di.fc.ul.pt
}

\author{
Francesca De Simone \\ Centrum Wiskunde \& Informatica \\ Amsterdam, The Netherlands \\ f.de.simone@cwi.nl
}

\author{
Rene Kaiser \\ Know-Center \\ Graz, Austria \\ rkaiser@know-center.at
}

\author{
Nimesha Ranasinghe \\ School of Computing and Information Science \\ University of Maine \\ USA \\ r.ranasinghe@maine.edu
}

\author{
Wendy Van den Broeck \\ imec-smit \\ Vrije Universiteit Brussel \\ Brussels, Belgium \\ Wendy.van.den.broeck@vub.be
}

\begin{abstract}
AltMM 2018 is the $3^{\text {rd }}$ edition of the International Workshop on Multimedia Alternate Realities at ACM Multimedia. Our ambition remains to engage researchers and practitioners in discussions on how we can successfully create meaningful multimedia 'alternate realities' experiences. One of the main strengths of this workshop is that we combine different perspectives to explore how the synergy between multimedia technologies can foster and shape the creation of alternate realities and make their access an enriching and valuable experience.
\end{abstract}

\section{CCS CONCEPTS}

- Human-centered computing $\rightarrow$ Virtual reality $\bullet$ Humancentered computing $\rightarrow$ Interactive systems and tools

\section{KEYWORDS}

Immersive Media; Interactive Multimedia; Mulsemedia; Multimedia Experience; Alternate Realities; Experiences; QoE.

\section{ACM Reference format:}

T. Chambel, F. De Simone, R. Kaiser, N. Ranasinghe and W. Van den Broeck. 2018. AltMM 2018 - 3rd International Workshop on Multimedia Alternate Realities. In Proceedings of ACM Multimedia conference (MM'18). ACM, NY, NY, USA, 2 pages. https://doi.org/10.1145/3240508.3243718
Permission to make digital or hard copies of all or part of this work for personal or classroom use is granted without fee provided that copies are not made or distributed for profit or commercial advantage and that copies bear this notice and the full citation on the first page. Copyrights for components of this work owned by others than ACM must be honored. Abstracting with credit is permitted. To copy otherwise, or republish, to post on servers or to redistribute to lists, requires prior specific permission and/or a fee. Request permissions from Permissions@acm.org. $M M$ '18, October 22-26, 2018, Seoul, Republic of Korea (c) 2018 Copyright is held by the owner/author(s).

ACM ISBN 978-1-4503-5665-7/18/10.

https://doi.org/10.1145/3240508.3243718

\section{INTRODUCTION}

Multimedia experiences allow us to access other worlds, to live other people's stories, to communicate with or experience alternate realities. Different spaces, times or situations can be entered thanks to multimedia contents and systems, which coexist with our current reality, and are sometimes so vivid and engaging that we feel we are living in them.

Today, different tools and technologies exist to create all sorts of interactive experiences for the user, trying to provide the ultimate alternate experience.

The traditional high-quality audiovisual media like TV and cinema, in combination with new tools such as head-mounted displays, $360^{\circ}$ cameras, haptic feedback and even smell and taste kits provide the potential to take the experience of alternate realities to a next level. But what kind of experiences are truly meaningful for the user? What are possible enablers and barriers for this type of experiences? These will be the topics addressed during the AltMM2018 workshop, the third edition of the series after a successful debut at ACM MM in Amsterdam, NL, in 2016 [1] and the second edition at ACM MM in Mountain View, USA in 2017 [2].

\section{MULTIMEDIA ALTERNATE REALITIES}

Alternate realness relates with the feelings of presence, engagement, flow and full immersion in a multimedia experience. This can be achieved, for example, through the production of immersive ultra-high quality and panoramic $/ 360^{\circ}$ content, and the consumption of media through immersive devices and environments (e.g. AR/VR, HMD and UHD displays, mulsemedia). But independent of the content format and the device on which it is consumed, content itself has the potential to be engaging and transport the user to an alternate reality. Users might live the story in a lean-backward setting or exploit interaction mechanisms to participate, shape and personalize the story and reality actively. The engagement in alternate realities might be particularly strong if the experience is shared with others, through means to communicate live, or capture to recount later. The extent to which 
multimedia content and systems facilitate the access of alternate realities is a measure of their effectiveness. So finally, we must have the means to design and evaluate our experience in alternate realities, with methods and measures to create and assess presence, engagement and full immersion.

The AltMM 2018 workshop aims at exploring how the synergy between multimedia technologies can foster the creation of alternate realities and make their access an enriching and valuable experience. We seek contributions that present multimedia technologies, methods and measurement approaches from the perspective of "enabling other realities". In particular, one or more of the following dimensions must be addressed in the contributions by prospective authors, when characterizing the type of multimedia alternate realities that they are aiming for:

- $\quad$ Alternate - refers to what is alternate about it: different space, time, situation, and so on;

- Virtual/Augmented - how far or close to the actual reality content can be experienced, ranging from totally virtual to augmented reality (VR/AR);

- Real/Fictional - how real or fictional the content is;

- Interactive - the level of interactivity as a means of engagement and immersion;

- Immersive - level in perceptual, cognitive and emotional terms, the sense of presence and belonging, the quality of the content and the experience, imagination and engagement;

- Multisensorial - the media involved and how much mulsemedia it is, also going beyond audiovisual content to include the five senses;

- Personal - adaptation to individual preferences and contexts;

Social - individualized vs shared experiences and communication.

These dimensions will be further complemented with other topics in the different contributions.

\section{TOPICS}

AltMM 2018 welcomed contributions with the goals and the perspective of enabling alternate realities experiences as characterized above, through multimedia technologies, and design and evaluation methods for its creation and consumption. This involves the use of different types of media content (audiovisual, haptics, smell, and taste), increased immersion (e.g. $3 \mathrm{D}$, holographic, UHD, $360^{\circ}$, and stereoscopic audio), new interaction devices, environments, modalities and formats.

\section{PROGRAM}

The workshop proposes a full-day program ${ }^{1}$ combining oral presentations, an invited keynote talk, poster and demo interactive presentations, and fishbowl like discussions, promoting the value of interactive discussions for effective knowledge exchange.
Our keynote speaker is Professor Woontack Woo (Graduate School of Cultural Technology (GSCT), Korea Advanced Institute of Science and Technology (KAIST)). His keynote talk on the topic of "Augmented Human: beyond augmented reality", will focus on the challenges of the co-existence of human, virtual and reality in the near future. He will share his view on how Augmented Human (AH) can broaden human's physical, intellectual and social capabilities.

The paper presentations will also stimulate the discussion on some interesting aspects of alternate multimedia realities. Topics will include innovative display and interaction techniques to deploy immersive multimedia experiences, via Head-Mounted Displays or Cave Automatic Virtual Environments; the design of virtual collaborative experiences, such as augmented reality tours or escape room games; the design of systems to add sensory modalities to alternate realities, for example by reproducing smells in addition to visual, audio and haptic modalities.

AltMM 2018 accepted 5 submissions of which 4 full and 1 short paper. All papers received 3-4 double blind peer reviews. The organisers explicitly want to thank the Program Committee members for their effort in reviewing and for their insightful assessments and comments. We also want to welcome all participants and encourage them to discuss and share new ideas on this though-provoking topic of Multimedia Alternate Realities.

\section{ACKNOWLEDGMENTS}

This workshop is supported by the project Power Semiconductor and Electronics Manufacturing 4.0 (SemI40), under grant agreement No 692466. The project is co-funded by grants from Austria, Germany, Italy, France, Portugal and Electronic Component Systems for European Leadership Joint Undertaking (ECSEL JU). The Know-Center is funded within the Austrian COMET Program (Competence Centers for Excellent Technologies) under the auspices of the Austrian Federal Ministry of Transport, Innovation and Technology, the Austrian Federal Ministry of Economy, Family and Youth and by the State of Styria. COMET is managed by the Austrian Research Promotion Agency FFG. The workshop is also partially supported by FCT through LASIGE funding ref.UID/CEC/00408/2013, and by FCT and MCE Portugal (PIDDAC) under grant UID/BIO/00645/2013.

\section{REFERENCES}

[1] Teresa Chambel, Rene Kaiser, Omar Niamut, Wei Tsang Ooi, and Judith A Redi. 2016. AltMM 2016: 1st International Workshop on Multimedia Alternate Realities. In Proceedings of the 2016 ACM on Multimedia Conference (MM'16). ACM, New York, NY, USA, 1481-1482. DOI: https://doi.org/10.1145/2964284.2980531

[2] Teresa Chambel, Rene Kaiser, Omar Niamut, and Wei Tsang Ooi. 2017. AltMM 2017: $2^{\text {nd }}$ International Workshop on Multimedia Alternate Realities. In Proceedings of the 2017 ACM on Multimedia Conference (MM '17). ACM, New York, NY, USA, 1969-1970. DOI: https://doi.org/10.1145/3123266.3132055

\footnotetext{
${ }^{1}$ http://altmm2018.di.fc.ul.pt/program.html
} 